\title{
Localization of dipeptidyl aminopeptidase yscIV in the plasma membrane of Saccharomyces cerevisiae
}

\author{
Carmen bordallo, Rosario Cueva and Paz Suárez Rendueles* \\ Area de Bioquimica y Biología Molecular, Departamento de Biología Funcional, Facultad de Medicina, \\ Universidad de Oviedo, E-33071 Oviedo, Spain
}

(Received 4 October 1989; accepted 5 December 1989)

\begin{abstract}
The subcellular distribution of dipeptidyl aminopeptidase activity was studied in protoplast lysates of Saccharomyces cerevisiae that were virtually free from vacuolar contamination. Dipeptidyl aminopeptidase yscIV, the STE13 gene product, was found to be associated with plasma membrane vesicles after sucrose gradient isopycnic centrifugation. Another dipeptidyl aminopeptidase activity, not yet fully characterized, was localized in a microvesicular population co-sedimenting with chitosomes.
\end{abstract}

\section{Introduction}

The yeast Saccharomyces cerevisiae contains a number of proteinases which play a vital role in cellular control (for recent reviews see Suárez-Rendueles \& Wolf, 1988; Hirsch et al., 1989).

We have previously described a membrane-bound Xprolyl dipeptidyl aminopeptidase activity in yeast (Suárez-Rendueles et al., 1981; García Ramos et al., 1983), later reported to be due to at least two different enzymes (Julius et al., 1983; Bordallo et al., 1984). One of the enzymes, which is inactivated by heating crude extracts at $60^{\circ} \mathrm{C}$ for $20 \mathrm{~min}$, was termed dipeptidyl aminopeptidase yscV. This enzyme is associated with vacuolar membranes (Bordallo et al., 1984) from which it was purified and characterized as a serine peptidase acting on proline-containing substrates (García-Alvarez et al., 1985). Genetic studies have shown that the gene designated $D A P 2$ is the structural gene for dipeptidyl aminopeptidase yscV, the biological role of which is unknown as dap 2 mutants do not show any obvious difference in phenotype (Suárez-Rendueles \& Wolf, 1987). The other enzyme, coded by the STE13 gene and termed dipeptidyl aminopeptidase yscIV is heat-resistant and is involved in the processing of the $\alpha$-factor pheromone precursor (Julius et al., 1983). MAT $\alpha$ ste13 mutant cells do not mate (Sprague et al., 1981) because they secrete incompletely processed forms of $\alpha$-factor due to the absence of dipeptidyl aminopeptidase yscIV (Julius et al., 1983).

Abbreviation: TEA, triethylamine.
The synthesis and processing of pre-pro- $\alpha$-factor have been studied with the aid of mutants blocked at various stages in the yeast secretory pathway (Julius et al., 1984). The first stage of proteolytic cleavage of the precursor into smaller units was detected in a mutant strain ( $\mathrm{sec} 7$ ) that accumulates Golgi-body-like structures at the restrictive temperature; processing is then completed within secretory vesicles (Julius et al., 1984). These findings led to the hypothesis that the specific proteinase responsible for the last processing step of $\alpha$-factor precursor, i.e. dipeptidyl aminopeptidase yscIV, is located in secretory vesicles, plasma membrane or both (Julius et al., 1984).

In this paper we describe the subcellular distribution of dipeptidyl aminopeptidase activity in $S$. cerevisiae lysates prepared by disrupting protoplasts in conditions minimizing organelle rupture.

\section{Methods}

Yeast strains and growth conditions. S. cerevisiae 1022 (ETH, Zurich, Switzerland; ATCC 32167), a wild-type strain kindly supplied by Dr A. Wiemken, and S. cerevisiae A2S3 (MATa ste13-1 ade6 leul trp5 his6 metl canl rmel gal2) (Sprague et al., 1981), defective in dipeptidyl aminopeptidase yscIV activity, were used.

Cells were grown in liquid mineral medium $(0.67 \%$ yeast nitrogen base without amino acids, $1 \%$, w/v, glucose) supplemented, when necessary, with the amino acids required by the auxotrophic strain. Cells were grown aerobically at $28^{\circ} \mathrm{C}$ and harvested at the exponential phase of growth.

Preparation, labelling and lysis of protoplasts. Protoplasts from $S$. cerevisiae 1022 were prepared and purified according to the method of Schwencke et al., 1983. When strain A2S 3 was used, exponential-phase 
yeast cells pre-treated as above were resuspended in $0.6 \mathrm{M}$-sorbitol, $10 \mathrm{~mm}$-EDTA, $20 \mathrm{~mm}$-MES/Tris (pH 6.0) containing $0.5 \%(\mathrm{w} / \mathrm{w})$ Zymolyase 20000 . Conversion to protoplasts was usually complete within $60 \mathrm{~min}$ with both strains. Two different methods of protoplast preparation were used because we found that the cell wall of strain A2S3 was more sensitive to Zymolyase than to Helicase.

Labelling and lysis of protoplasts were done as described by Flores Martínez \& Schwencke (1988). A suspension of protoplasts $\left(10^{9} \mathrm{ml}^{-1}\right)$ in $0.6 \mathrm{M}$-sorbitol, $10 \mathrm{mM}-\mathrm{MgCl}_{2}, 20 \mathrm{mM}$-MOPS/Tris, pH 7.0 (buffer A) was mixed with $0.25 \mu \mathrm{Ci}(9.25 \mathrm{kBq})\left[{ }^{3} \mathrm{H}\right]$ concanavalin $A$ dissolved in buffer A. After $10 \mathrm{~min}$ at $30^{\circ} \mathrm{C}$, protoplasts were collected by centrifugation (swinging-bucket rotor) at $3500 \mathrm{~g}$ for $5 \mathrm{~min}$ and washed twice with ice-cold buffer $A$. The pellet was carefully resuspended in 1 M-sorbitol, 25 mM-triethylamine (TEA)/HCl, pH 8.0, 1 mM-EDTA and kept on ice for $10 \mathrm{~min}$. The protoplast suspension was diluted $1: 4$ with pre-warmed $\left(30^{\circ} \mathrm{C}\right)$ disrupting buffer $(0.36 \mathrm{M}$-sorbitol, $25 \mathrm{~mm}$ TEA $/ \mathrm{HCl}, \mathrm{pH} 8 \cdot 0,50 \mathrm{~mm}$-glucose, $2 \cdot 5 \mathrm{~mm}-\mathrm{K}_{2} \mathrm{CO}_{3}$ ) and incubated for $10 \mathrm{~min}$ at $30^{\circ} \mathrm{C}$. Rupture of protoplasts and vacuole liberation was assessed by phase-contrast microscopy.

Isopycnic sucrose gradient centrifugation. The protoplast lysate was centrifuged at $500 \mathrm{~g}$ for $15 \mathrm{~min}$ (swinging-bucket rotor) to remove vacuoles, intact protoplasts and debris. The supernatant was filtered through Sepharose-2B as described by Flores Martinez \& Schwencke (1988). Void volume fractions were pooled, sucrose was added to adjust the concentration to $20 \%(\mathrm{w} / \mathrm{w})$ and used in conjunction with a $60 \%$ (w/w) sucrose solution to construct a $20-60 \%$ (w/w) linear sucrose gradient $(32 \mathrm{ml})$. The gradients were centrifuged in a Beckman SW-25 rotor at $70000 \mathrm{~g}\left(\mathrm{r}_{\mathrm{av}}, 9 \cdot 27 \mathrm{~cm}\right)$ for $40 \mathrm{~h}$. Fractions $(1 \mathrm{ml})$ were collected from the bottom upwards by means of a peristaltic pump and analysed for enzyme activities as described below. Density was determined by refractometry.

Enzyme assays. Dipeptidyl aminopeptidase activity was determined with Ala-Pro-4-nitroanilide as substrate as described previously (Suárez-Rendueles et al., 1981). Chitin synthetase was assayed according to Ruiz-Herrera \& Bartnicki-García (1976), but using $60 \mu \mathrm{g}$ $\alpha$-chymotrypsin $\mathrm{ml}^{-1}$ to activate zymogenic chitin synthetase as indicated by Flores Martínez \& Schwencke (1988). Carboxypeptidase yscY activity was assayed by the method of Aibara et al. (1971) with benzoyl-tyrosine-4-nitroanilide as substrate; as a modification, $0.5 \%$ sodium deoxycholate was added (Bordallo et al., 1984). $\alpha$-Mannosidase activity was determined with $p$-nitrophenyl $\alpha$-D-mannopyranoside as substrate as described by Opheim (1978).

Protein determination. Protein was determined according to the method of Bradford (1976).

Substrates and chemicals. Ala-Pro-4-nitroanilide was supplied by Bachem, Switzerland. Crystallized $\alpha$-chymotrypsin was purchased from Serva. Uridine diphospho- $N$-acetyl-D-[U-14 $\mathrm{C}]$ glucosamine [ammonium salt; $\left.247 \mathrm{mCi} \mathrm{mmol}^{-1}\left(9 \cdot 14 \mathrm{GBq} \mathrm{mmol}^{-1}\right)\right]$ and $N$-acetyl$\left[{ }^{3} \mathrm{H}\right]$ concanavalin A $\left[47.6 \mathrm{Ci} \mathrm{mmol}^{-1}\left(1.76 \mathrm{TBq} \mathrm{mmol}^{-1}\right)\right]$ were obtained from Amersham. Sepharose-2B was from Pharmacia. All other chemicals, which were of the highest purity available, were purchased from either Merck or Sigma. Zymolyase was obtained from Kirin, Japan.

\section{Results}

\section{Subcellular distribution of dipeptidyl aminopeptidase yscIV}

The use of a mild disruption procedure for yeast protoplasts, as described by Flores Martínez \&

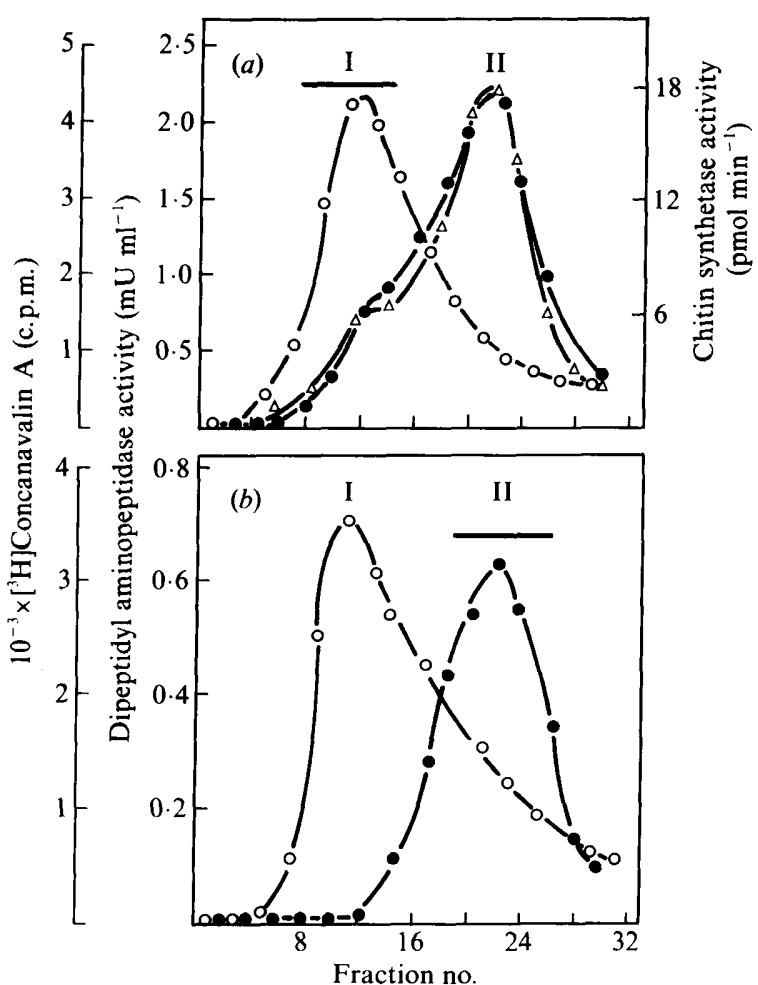

Fig. 1. Isopycnic sucrose gradient centrifugation of a partially purified vesicular fraction of $S$. cerevisiae. The contents of the void volume eluate of a Sepharose-2B column, obtained as described in Methods, were separated by centrifugation on a $20-60 \%(w / w)$ linear sucrose gradient. Tubes were centrifuged at $70000 \mathrm{~g}\left(\mathrm{r}_{\mathrm{av}} .9 \cdot 27 \mathrm{~cm}\right)$ for $40 \mathrm{~h}$. (a) strain 1022 (wild-type); (b) strain A2S3 (ste13). O, $\left[{ }^{3} \mathrm{H}\right]$ Concanavalin A; $\triangle$, chitin synthetase activity; $\bigcirc$, dipeptidyl aminopeptidase activity.

Schwencke (1988), allowed us to investigate the subcellular localization of the membrane-bound dipeptidyl aminopeptidase yscIV free from the interference caused by dipeptidyl aminopeptidase yscV, an enzyme with overlapping substrate specificity, which is located in the vacuolar membrane (Bordallo et al., 1984). Protoplasts from the wild-type strain $S$. cerevisiae 1022 were labelled with $\left[{ }^{3} \mathrm{H}\right]$ concanavalin $\mathrm{A}$ and lysed as indicated in Methods. After filtration through Sepharose-2B, the particulate fraction eluting in the void volume of the column was subjected to isopycnic sucrose gradient centrifugation $(20-60 \%$, w/w, sucrose); two peaks of dipeptidyl aminopeptidase activity were reproducibly obtained (Fig. 1a). The main peak (peak II) contained vesicles having a buoyant density $\left(1.145 \mathrm{~g} \mathrm{~cm}^{-3}\right)$ typical of chitosomes (Bracker et al., 1976; Ruiz-Herrera et al., 1984; Flores Martínez \& Schwencke, 1988). The other peak (peak I) contained plasma membrane vesicles, identified on the basis of their buoyant density $(1.203 \mathrm{~g}$ $\left.\mathrm{cm}^{-3}\right)$ and by the fact that the $\left[{ }^{3} \mathrm{H}\right]$ concanavalin $\mathrm{A}$ cosedimented with them (Scarborough, 1975; Boller et al., 1976; Flores Martínez \& Schwencke, 1988). 
Further evidence for the identity of the two fractions was obtained by measuring chitin synthetase activity along the gradient (Fig. 1a). The distribution of chitin synthetase was similar to that described above for dipeptidyl aminopeptidase. In both peaks chitin synthetase was present mostly in zymogen form: without proteolytic activation by chymotrypsin less than $5 \%$ of the total chitin synthetase activity could be measured. The same results concerning zymogenic chitin synthetase distribution were reported by Flores Martínez \& Schwencke (1988) using the same yeast strain. These authors identify peak $I$ as plasma membrane particles and peak II as chitosome vesicles. In agreement with those authors we were unable to detect carboxypeptidase yscY activity (a soluble vacuolar-enzyme marker) in either the material excluded from the Sepharose-2B column or in the fractions collected after the isopycnic sucrose gradient centrifugation, thus ruling out the possibility of interference by soluble vacuolar proteinases. We also assayed $\alpha$-mannosidase activity as a measure of cross-contamination by vacuolar membranes: about $15 \%$ of the total $\alpha$-mannosidase activity remained in the $500 \mathrm{~g}$ supernatant after protoplast lysis. However, no $\alpha$-mannosidase activity could be detected after filtration through the Sepharose-2B column, as this process inactivated the enzyme for unknown reasons. Therefore, the possibility of a low level of contamination of the gradient fractions by vacuolar membranes and hence by the tonoplast-bound dipeptidyl aminopeptidase yscV could not be ruled out completely.

Preliminary evidence that dipeptidyl aminopeptidase yscIV, the enzyme responsible for $\alpha$-factor precursor processing, was the activity detected in plasma membrane vesicles (Fig. 1 $a$, peak I) came from kinetic studies. No significant loss of activity was observed after heating gradient fractions (under the bar in peak I, Fig. 1 a) at $60^{\circ} \mathrm{C}$ for $20 \mathrm{~min}$. This behaviour is characteristic of dipeptidyl aminopeptidase yscIV (Julius et al., 1983; Bordallo et al., 1984). Also, the affinity for the substrate Ala-Pro-4-nitroanilide was very similar to that reported for dipeptidyl aminopeptidase yscIV (Bordallo et al., 1984), the apparent $K_{\mathrm{m}}$ value being $0.9 \mathrm{~mm}$ (data not shown). Further evidence that dipeptidyl aminopeptidase yscIV is bound to the plasma membrane was obtained by using a yeast strain devoid of dipeptidyl aminopeptidase yscIV activity due to a single chromosomal gene mutation affecting its structural gene (STE13) (Julius et al., 1984; Suárez-Rendueles \& Wolf, 1987). Protoplasts from strain A2S3 (ste13) were labelled with $\left[{ }^{3} \mathrm{H}\right]$ concanavalin $\mathrm{A}$, lysed and filtered through a Sepharose-2B column following the protocol used for the wild-type strain. When the void volume fractions from this column were subjected to isopycnic sucrose gradient centrifugation $(20-60 \%, w / w)$, the distribution of dipep-

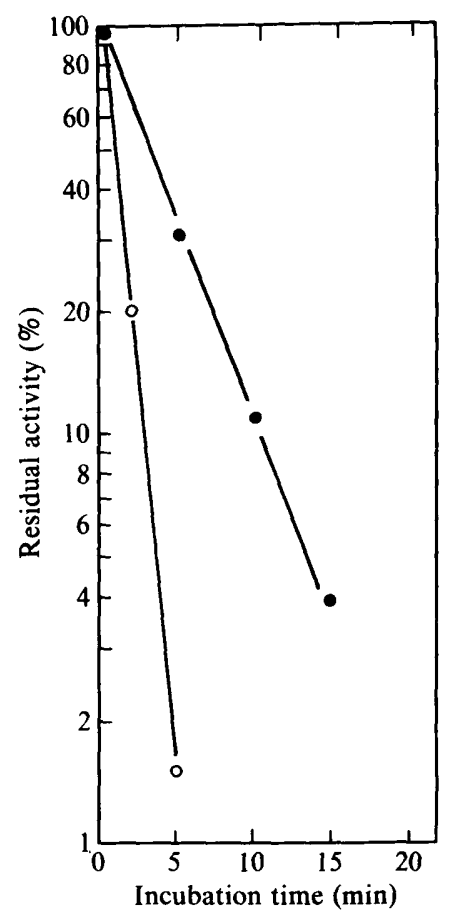

Fig. 2. Thermal inactivation of dipeptidyl aminopeptidase activity. Samples prepared from $S$. cerevisiae A2S3 (stel3), were incubated in $0.1 \mathrm{M}$-Tris/ $\mathrm{HCl}$ buffer, $\mathrm{pH} 7.0$, for the times indicated, quickly cooled and equilibrated at $37^{\circ} \mathrm{C}$ for $5 \mathrm{~min}$ before assay. The enzyme activity remaining was measured as described in Methods. Whole membrane fraction; $O$, chitosomes (dialysed pooled fractions indicated by the bar in Fig. 1b).

tidyl aminopeptidase activity was as shown in Fig. 1(b). Only the peak of activity (peak II) at specific gravity $1 \cdot 145 \mathrm{~g} \mathrm{~cm}^{-3}$ (chitosomes) was detected, confirming that the plasma-membrane-bound activity detected in the wild-type strain was due to dipeptidyl aminopeptidase yscIV.

Characterization of the dipeptidyl aminopeptidase activity co-sedimenting with chitosomes

The thermal stability of dipeptidyl aminopeptidase activity was measured both in a whole membrane fraction (prepared as described by Suárez-Rendueles $e t$ $a l ., 1981)$ and in purified chitosomes prepared from the mutant strain lacking dipeptidyl aminopeptidase yscIV (fractions under the bar in Fig. $1 b$ ). As expected, in the whole membrane fraction a heat-labile dipeptidyl aminopeptidase activity, corresponding mainly to dipeptidyl aminopeptidase yscV (Bordallo et al., 1984), was detected (Fig. 2). A quite different picture was obtained when the heat stability of chitosome-bound activity was studied. Heating chitosomes at $60^{\circ} \mathrm{C}$ for 5 min inactivat- 


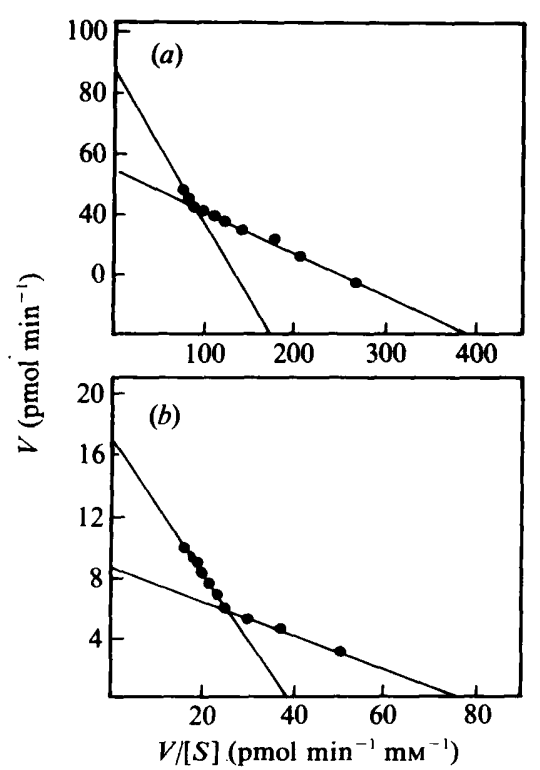

Fig. 3. Eadie-Hofstee plot of dipeptidyl aminopeptidase activity with Ala-Pro-4-nitroanilide as substrate in samples prepared from $S$. cerevisiae A2S3 (ste13). Enzyme activity was assayed at $37^{\circ} \mathrm{C}$ as outlined in Methods. (a) Whole membrane fraction; (b) chitosomes (dialysed pooled fractions indicated by the bar in Fig. $1 b$ ).

ed chitosomal dipeptidyl aminopeptidase activity completely (Fig. 2), whereas dipeptidyl aminopeptidase yscV retains almost $50 \%$ activity under such conditions (Bordallo et al., 1984).

The affinity for the substrate Ala-Pro-4-nitroanilide was determined using either a whole membrane fraction or purified chitosomes from strain $\mathrm{A} 2 \mathrm{~S} 3$ as enzyme sources (Fig. 3). Two components with different substrate affinities $\left(K_{\mathrm{m}}\right.$ values 0.09 and $\left.0.44 \mathrm{mM}\right)$ were present in both membrane preparations but their relative contribution to the total dipeptidyl aminopeptidase activity measured depended strongly on the enzyme source. Thus, dipeptidyl aminopeptidase yscV $\left(K_{\mathrm{m}}\right.$ $0.09 \mathrm{mM}$ ) (Bordallo et al., 1984) was the major activity detected in the whole membrane preparation (Fig. $3 a$ ), while a different enzyme, with lower affinity for the substrate $\left(K_{\mathrm{m}} 0.44 \mathrm{mM}\right)$ was the major component in the chitosome vesicles (Fig. 3 b). Dipeptidyl aminopeptidase yscV and the activity co-sedimenting with chitosomes could not be distinguished by sensitivity to inhibitors or isoelectric point. Both activities were completely inhibited by PMSF, a specific inhibitor of serine proteinases, and by various divalent cations such as $\mathrm{Hg}^{2+}, \mathrm{Cd}^{2+}, \mathrm{Zn}^{2+}$ and $\mathrm{Ni}^{2+}$. The isoelectric point determined by isoelectric focusing in the $\mathrm{pH}$ range 4-6.5 using a glycerol gradient showed that both activity peaks had a pI of 4.9 (GarciaAlvarez et al., 1985).

\section{Discussion}

Determining the subcellular distribution of dipeptidyl aminopeptidase yscIV was difficult for two reasons. First, in the wild-type strain the dipeptidyl aminopeptidase yscV, which has overlapping substrate specificity, accounts for approximately $70 \%$ of the total activity measurable in a protoplast lysate (Julius et al., 1983; Bordallo et al., 1984). Secondly, obtaining pure preparations of yeast cell organelles, is difficult, especially when harsh methods of cell disruption are used.

The recent publication of a mild iso-osmotic disruption method of yeast protoplasts that leaves most of the vacuoles intact and minimizes cross-contamination of subcellular fractions (Flores Martínez \& Schwencke, 1988 ), allowed us to study the location of dipeptidyl aminopeptidase yscIV. The physiological role ( $\alpha$-factor precursor processing) of this enzyme indicates that it could be located in the plasma membrane, in secretory vesicles or in both (Julius et al., 1984).

The results presented in this paper show that dipeptidyl aminopeptidase activity from a wild-type strain is associated with two different particulate fractions after isopycnic sucrose gradient centrifugation of a partially purified protoplast lysate from which most vacuoles and thus most dipeptidyl aminopeptidase yscV activity had been eliminated. The fraction equilibrating at a buoyant density of $1.203 \mathrm{~g} \mathrm{~cm}^{-3}$ and containing the bulk of bound $\left[{ }^{3} \mathrm{H}\right]$ concanavalin $\mathrm{A}$ has been identified by several criteria as plasma-membrane ghost vesicles by Flores Martínez \& Schwencke (1988) who used protoplast homogenates from the same yeast strain. The other fraction, equilibrating at a lower buoyant density $\left(1 \cdot 145 \mathrm{~g} \mathrm{~cm}^{-3}\right)$ has been characterized as chitosomes by these authors. It must be kept in mind, however, that other vesicular material such as proteasomes (Tanaka $e t$ $a l ., 1988)$ may sediment in the same fraction. Nevertheless, the proteolytic activity detected so far in proteasomes shows latency, while the dipeptidyl aminopeptidase activity described here can be detected without previous activation.

The data presented clearly demonstrate that dipeptidyl aminopeptidase activity associated with plasmamembrane vesicles is absent in a yeast strain defective in dipeptidyl aminopeptidase yscIV due to a single chromosomal mutation (ste13), strongly supporting the proposal that this enzyme is localized at the yeast plasma membrane. The fact that we have been unable to detect dipeptidyl aminopeptidase activity in whole cells that have not first been permeabilized to permit entry of the small peptide substrate used for the assay probably means that dipeptidyl aminopeptidase yscIV is not an exocellular plasma membrane protein.

Dipeptidyl aminopeptidase activity co-sedimenting 
with chitosomes was found both in a wild-type yeast strain and in the ste13 mutant strain. This shows that chitosomal dipeptidyl aminopeptidase activity cannot be due to dipeptidyl aminopeptidase yscIV. The preliminary data presented here concerning the identification and characterization of activity sedimenting with the chitosomal fraction suggest that although there is some vacuolar contamination of the chitosomal fraction, as judged by the presence of dipeptidyl aminopeptidase yscV activity, most of the activity co-sedimenting with these microvesicles might be due to a different enzyme. Further studies are in progress in order to identify and better characterize this activity.

The relative distribution of dipeptidyl aminopeptidase activity between the plasma membrane and the chitosomes found in the study probably does not exactly reflect the situation 'in vivo', because of the possible fusion of plasma membrane and chitosomes which can occur after cell disruption and during the purification, and because the chitosomal fraction was slightly contaminated with vacuolar dipeptidyl aminopeptidase yscV.

We conclude that the product of the STE13 gene, dipeptidyl aminopeptidase yscIV, is localized at the plasma membrane, and that another dipeptidyl aminopeptidase activity, not yet characterized, is localized in a vesicular fraction co-sedimenting with chitosomes.

This work was supported by grant $2582 / 83$ from the Comision Asesora para la Investigación Científica y Técnica of Spain. We extend special thanks to Jaime Schwencke for providing information prior to publication.

\section{References}

Aibara, S., Hayashi, R. \& Hata, T. (1971). Physical and chemical properties of yeast proteinase C. Agricultural and Biological Chemistry 35, 658-666.

BOLLER, T., DÜrR, M. \& WIEMKen, A. (1976). Asymmetric distribution of concanavalin $\mathrm{A}$ binding sites on yeast plasmalemma and vacuolar membrane. Archives of Microbiology 109, 115-118.

Bordallo, C., SChWEnCKe, J. \& SuÁrez-Rendueles, M. P. (1984). Localization of the thermosensitive X-prolyl dipeptidyl aminopeptidase in the vacuolar membrane of Saccharomyces cerevisiae. FEBS Letters 173, 199-203.

Bracker, C. E., Ruiz-Herrera, J. \& Bartnicki-García, S. (1976). Structure and conformation of chitin synthetase particles (chitosomes) during microfibril synthesis in vitro. Proceedings of the National Academy of Sciences of the United States of America 73, 4570-4574.
BRADFORD, M. M. (1976) A rapid and sensitive method for the quantitation of microgram quantities of protein utilizing the principle of protein-dye binding. Analytical Biochemistry 72, 248254.

Flores Martínez, A. \& SCHWencke, J. (1988). Chitin synthetase activity is bound to chitosomes and to the plasma membrane in protoplasts of Saccharomyces cerevisiae. Biochimica et Biophysica Acta 946, 328-336.

García-Alvarez, N., Bordallo, C., Gascón, S. and SuárezRendueles, P. (1985). Purification and characterization of a thermosensitive $X$-prolyl dipeptidyl aminopeptidase (dipeptidyl aminopeptidase yscV) from Saccharomyces cerevisiae. Biochimica et Biophysica Acta 832, 119-125.

García Ramos, C., Cruz Camarillo, R., Gascón, S. \& Suárez RENDUELES, M. P. (1983). Studies on the regulation of X-prolyl dipeptidyl aminopeptidase activity. Journal of General Microbiology 129, 3519-3523.

Hirsch, H. H., Suárez-Rendueles, P. \& Wolf, D. H. (1989). Yeast Saccharomyces cerevisiae proteinases. Structure, characteristics and function. In The Molecular and Cell Biology of Yeasts, pp. 134-200. Edited by E. F. Walton \& G. T. Yarranton. Glasgow: Blackie.

Julius, D., Blair, L., Brake, A., Sprague, G. \& Thorner, J. (1983). Yeast $\alpha$-factor is processed from a larger precursor polypeptide: the essential role of a membrane-bound dipeptidyl aminopeptidase. Cell $32,839-852$.

Julius, D., Schekman, R. \& Thorner, J. (1984). Glycosylation and processing of prepro- $\alpha$-factor through the yeast secretory pathway. Cell 36, 309-318.

OPHEIM, D. S. (1978). $\alpha$-Mannosidase of Saccharomyces cerevisiae. Characterization and modulation of activity. Biochimica et Biophysica Acta 524, 121-130.

Ruiz-Herrera, J. \& Bartnicki-García, S. (1976). Proteolytic activation and inactivation of chitin synthetase from Mucor rouxii. Journal of General Microbiology 97, 241-249.

Ruiz-Herrera, J., Bracker, C. E. \& BartNicki-García, S. (1984). Sedimentation properties of chitosomes from Mucor rouxii. Protoplasma 122, 176-190.

SCARBOROUGH, G. A. (1975). Isolation and characterization of Neurospora crassa plasma-membrane. Journal of Biological Chemistry 250, $1106-1111$.

SChWEncke, J., Canut, H. \& Flores, A. (1983). Simultaneous isolation of the yeast cytosol and well-preserved mitochondria with negligible contamination by vacuolar proteinases. FEBS Letters 156 , 274-280.

SPRAGUe, G. F., JR, Rine, J. \& HersKowitz, I. (1981). Control of yeast cell type by the mating type locus. II. Genetic interactions between MAT and unlinked $\alpha$-specific STE genes. Journal of Molecular Biology 153, 323-335.

SuÁREZ-Rendueles, P. \& Wolf, D. H. (1987). Identification of the structural gene for dipeptidyl aminopeptidae yscV $(D A P 2)$ of Saccharomyces cerevisiae. Journal of Bacteriology 169, 404I-4048.

SuÁreZ-Rendueles, P. \& WolF, D. H. (1988). Proteinase function in yeast: biochemical and genetic approaches to a central mechanism of post-translational control in the eukaryotic cell. FEMS Microbiology Reviews 54, 17-46.

Suárez-Rendueles, M. P., Schwencke, J., García-Alvarez, N. \& Gascón, S. (1981). A new X-prolyl dipeptidyl aminopeptidase from yeast associated with a particulate fraction. FEBS Letters 131, 296300.

Tanaka, K., Yoshimura, T., Kumatori, A., Ichihara, A., Ikal, A., Nishigai, M., Kameyama, K. \& Takagi, T. (1988). Proteasomes (multi-protease complexes) as $20 \mathrm{~S}$ ring-shaped particles in a variety of eukaryotic cells. Journal of Biological Chemistry 263, 16209-16217. 